\title{
Las expectativas de los estudiantes y la calidad del servicio ofertado por la universidad de Sevilla
}

\section{Student's expectations and quality of services offered by University of Sevilla}

\author{
Rosario ORDÓÑEZ-SIERRA y Margarita R. RODRÍGUEZ-GALLEGO \\ Universidad de Sevilla
}

Recibido: Noviembre 2014

Evaluado: Mayo 2015

Aceptado: Mayo 2015

\section{Resumen}

En este artículo se presenta un análisis diferencial de las expectativas de los estudiantes de la Universidad de Sevilla (España), en función de las ramas del conocimiento, agrupadas en las dimensiones personal, universidad, curricular y docencia. Las aportaciones han sido recogidas en un cuestionario aplicado a 1082 estudiantes de las áreas de humanidades, bio-sanitaria, económica-jurídica y científico-técnica, a través de un análisis de la varianza para la comparación de medias (asumiendo varianzas iguales y pruebas de Scheffé y Tukey). Se han contrastado los valores medios de cada rama, con el fin de determinar si las diferencias observadas resultan estadísticamente significativas. Los resultados obtenidos ponen de manifiesto que la dimensión personal y la de universidad son valoradas de forma similar en las cuatro ramas del conocimiento; en cambio en la dimensión curricular, las expectativas son más favorables para la rama bio-sanitaria y, en la docencia para la rama de humanidades. En definitiva, los resultados presentan las fortalezas y las debilidades en las dimensiones consideradas y permiten extraer conclusiones de las que derivan indicadores de interés para la mejora de la comunidad universitaria.

Palabas clave: expectativas, educación superior, docencia, estudiantes, currículum.

\begin{abstract}
The aim of this article is to present a differential analysis about the expectations of students from the University of Seville (Spain), according to branches of knowledge, grouped in personal, university, curriculum, and teaching dimensions. Contributions have been collected from a questionnaire administered to 1082 students from the areas of humanities, bio-medical, economic-legal and scientific-technical, through an analysis of variance to compare average (assuming equal variances and Scheffé and Tukey tests). Average values of each branch have been contrasted, in order to determine whether the observed differences are statistically significant. The results obtained show that the personal and university dimensions are valued in a similar way in the four branches of knowledge, while the curriculum dimension received a higher value in degrees related with bio-medical, and teaching received a higher value in degrees of humanities. Finally, results show strengths and weaknesses in the considered
\end{abstract}


dimensions and allow obtaining conclusions from which it is possible to derive indicators of interest for the improvement of the university community.

Keywords: expectations, high education, teaching, students, curriculum.

Somos conscientes que en la formación y el proceso de desarrollo de las expectativas intervienen gran cantidad de variables que difieren de una persona a otra y de una situación a otra, por tanto, el efecto real de tales expectativas es difícil de generalizar. Por lo que en esta investigación nos hemos marcado como objetivo analizar las expectativas mostradas por los estudiantes sobre la eficiencia de los servicios ofertados por cada Universidad.

Al revisar la literatura, sobre el tema objeto de estudio, se comprueba que en el contexto español destacan las de Fernández Pérez (1989), Tejedor (1998, 2003), Trillo y Porto (2002) y Porto (2006), Steinmann, Bosch y Aiassa (2013) de ahí el motivo de este análisis.

Desde un planteamiento general, se entiende por expectativa una suposición centrada en el futuro realista o no (Cosacov, 2007) y la percepción anticipada de lo que sucederá en el futuro (Merhi, 2011). En el ámbito universitario, implica la decisión de qué titulación cursar, qué se espera de la Universidad, del proceso de enseñanzaaprendizaje, de la participación en las tareas de políticas, gestión o investigación. En este mismo sentido, Alonso (1999) estima que la capacidad del estudiante para realizar anticipaciones de futuro hace que desarrolle expectativas, y éstas se concreten en el uso del sistema de formación (el grado de servicio y fiabilidad esperada, las preferencias, intereses, etc.), siendo expectativas que el estudiante mantendrá en un momento dado en relación con los servicios universitarios.

En esta investigación, se han comparado en la dimensión personal las expectativas con respecto a la universidad, grado de interés por las actividades culturales y campañas de sensibilización sobre determinados temas sociales. En la dimensión universidad se han agrupado ítems sobre las expectativas de los estudiantes respecto al tipo de estudios, prácticas y salidas profesionales, los servicios e información que la Universidad les presta, actividades que oferta y sus posibilidades de participación en los Órganos de Gobierno; al considerarse que los estudiantes al ingresar en la universidad entran con diferentes expectativas respecto al tipo de educación y al futuro laboral que tendrán (Pichardo, García Berbén, De la Fuente y Justicia, 2007). La implicación de los estudiantes en el gobierno y funcionamiento de las Universidades ha de ser un objetivo explícito y visible en estas instituciones, no sólo porque gran parte de las decisiones tomadas en el gobierno de la Universidad afectan directamente a los estudiantes sino porque es la forma más natural de reconocer la importancia formativa y efectiva de estos procesos participativos (Benedicto y Morán, 1999; Eliophotou, 2003; Lizzio y Wilson, 2009 y Zuo y Rastoy, 1999).

En la dimensión curricular se han estudiado aspectos relativos a los objetivos, contenidos, actividades, metodología, medios o recursos empleados en el aula y evaluación. Siendo este último objeto de estudio en múltiples investigaciones (De la Orden, 2009; López, Cannella y Ciancimino, 2010; Sánchez, 2010 y Ricoy y 
Fernández-Rodríguez, 2013), pues la tarea de evaluar es evidentemente una actividad ardua y, compleja para el profesorado, que requiere de la valoración del desarrollo global del proceso y de sus participantes, lo que exige la utilización de distintas estrategias y procedimientos. En todo caso, se ha considerado que la evaluación ha de ser un medio para ayudar al profesorado a transformar la enseñanza o, al menos, para introducir cambios que contribuyan a mejorar la calidad del proceso de enseñanzaaprendizaje. Ésta no debe cubrir solo las exigencias que conllevan la adaptación de la docencia al Espacio Europeo de Educación Superior, sino que es imprescindible ir más allá de la transmisión de conceptos y la repetición de informaciones, contemplando la obtención de diferentes competencias, habilidades y destrezas y el desarrollo de su evaluación (Aguaded y López, 2009).

En la dimensión docencia se ha analizado la personalidad, tarea, relación interpersonal con los estudiantes y su formación profesional y humana. Actualmente, las evaluaciones de los estudiantes universitarios sobre la docencia de los profesores son un indicador de calidad docente muy utilizado (García Garduño y Medécigo Shej, 2014; Rodríguez-Ayán, 2005; Worthington, 2002). Son muchos los investigadores que dedican sus esfuerzos a indagar sobre cómo mejorar los procesos de enseñanzaaprendizaje desde diferentes perspectivas, centradas en el sujeto que aprende (León y Latas, 2005), en el contexto del aula (Entwistle y Tait, 1990), en el marco institucional (Ramsden, Martín y Boudén, 1989) o en el currículum (Gimeno, 2008). Dada la enorme relevancia de la figura del docente, nos preocupa e interesa conocer cuáles han de ser las características que lo definan para elaborar un perfil docente de calidad. La revisión de estudios realizados dentro de este campo ponen claramente de manifiesto que sería muy difícil alcanzar un perfil único, ya que éste dependerá de multitud de variables y condicionantes, entre los que cabría destacar tanto la metodología empleada como la fuente de la que obtuviésemos la información. Algunos autores han planteado perfiles no siempre concordantes (Apodaca y Grad, 2002; Beltrán, 2000; Ibáñez, 1990 y González y Fuentes, 1997), que evidencian que la diversidad de planteamientos que podemos encontrar complica la pretensión de establecer un perfil único. Por eso, en nuestro trabajo hemos acotado el campo de estudio analizando en qué medida las cualidades del profesor satisfacen las expectativas del alumnado.

Estas apreciaciones coinciden con la investigación realizada por Martínez García, García Domingo y Quintanal Díaz (2006) en la que plantea que el perfil del docente debe centrarse en las características, cualidades, condición, etc., que en opinión de los estudiantes universitarios deben presentar sus profesores en los distintos ámbitos de su actuación docente -pedagógica, investigadora, de gestión y personal-, conocimiento que servirá como vehículo de reflexión y retroalimentación que favorezca el desarrollo de nuestra labor profesional.

Para Salvador (2005: 184) "una de las metas primordiales de la enseñanza universitaria es considerar que el profesor ocupa un papel fundamental como referente central de todos los procesos educativos que tienen lugar en el aula y fuera de ella". Por ello, hacemos alusión a la docencia y, especialmente, a las características que debe poseer un profesor ideal: su personalidad; la materia que enseña; los métodos de 
enseñanza que desarrolla; su relación interpersonal con los alumnos; la evaluación de sus alumnos y su participación en la vida académica y comunitaria.

Debemos tener presente que estamos inmersos en un periodo de profundos cambios en la Universidad que van a afectar no sólo a la estructura de las diferentes titulaciones, sino también al contenido y la metodología de la misma docencia y formación universitaria es, pues, un buen momento para considerar qué papel queremos que tenga el estudiante dentro de nuestra Universidad y cuáles son los espacios, procesos y medios de formación que ponemos a su alcance para que desarrolle las competencias que se consideran deseables (Soler, Pallisera, Planas, Fullana y Vilá, 2012). El proceso de cambio por el que pasa la Universidad debe venir marcado, tal y como indica Salvador (2005: 184), por la calidad y, "una de las opciones a la hora de evaluar la calidad del servicio universitario se basa en la satisfacción de las expectativas de los usuarios", ya que la satisfacción con la formación recibida en la Universidad es uno de los aspectos de mayor presencia en la larga tradición de encuestas a egresados con que cuentan otros países.

\section{Método}

\section{Objetivo}

El objetivo general de este estudio es analizar las expectativas, en función de las ramas del conocimiento, mostradas por los estudiantes de la Universidad de Sevilla durante el curso académico 2012-2013.

\section{Participantes}

Para la selección de la muestra nos hemos basado en Downie y Heat (1983), utilizando un procedimiento de muestreo aleatorio estratificado, considerando como primer criterio las cuatro ramas del conocimiento de la Universidad de Sevilla (humanidades, bio-sanitaria, económica-jurídica y científico-técnica) y, como segundo criterio de selección, las diferentes titulaciones de dicha Universidad.

Para la elección de los individuos que constituirían las muestras se han estimado conglomerados, es decir, agrupamientos de turno mañana y tarde que de forma natural encontramos ya configurados en las respectivas poblaciones.

La muestra de estudiantes se ha extraído de forma aleatoria en cada Facultad dentro del número de centros aportado a la muestra por cada estrato poblacional (ramas del conocimiento, áreas y titulaciones), teniendo en cuenta el tamaño de los mismos.

La población elegible estuvo formada por estudiantes de la universidad de Sevilla, matriculados en el curso académico 2012-2013. El total de la población fue de 57.624 estudiantes, al tener una población tan extensa se ha realizado un muestreo aleatorio. Finalmente, la muestra que ha participado en esta investigación asciende a un total de 1082 sujetos; de los cuales el 69,1\% son mujeres, frente al 30,9\% que son hombres. El peso de respuesta de la población es mayor en las ramas de humanidades $(31 \%)$, bio- 
sanitarias $(36,9 \%)$ y económico-jurídicas $(25,1 \%)$, frente al $7 \%$ de la rama científicotécnica debido a la negativa del profesorado a formar parte de la investigación.

Tal y como se observa en la Tabla 1, presentamos las distintas Facultades agrupadas en función de las ramas del conocimiento y sus respectivos porcentajes de participación.

\begin{tabular}{llc}
\hline Ramas de conocimiento & Facultades & \% \\
\hline Humanidades & Fac. de Bellas Artes & 4.8 \\
\cline { 2 - 3 } & Fac. de Ciencias de la Educación & 19.4 \\
\cline { 2 - 3 } & Fac. de Comunicación & 2.6 \\
\cline { 2 - 3 } & Fac. de Filología & 4.2 \\
\hline \multirow{2}{*}{ Bio-Sanitaria } & Fac. de Enfermería, Fisioterapia y Podología & 8.5 \\
\cline { 2 - 3 } & Fac. de Farmacia & 11.1 \\
\cline { 2 - 3 } & Fac. de Medicina & 3.5 \\
\cline { 2 - 3 } & Fac. de Psicología & 12 \\
\cline { 2 - 3 } & Fac. de Odontología & 1.7 \\
\hline Económica-Jurídica & Fac. de Ciencias del Trabajo & 5.5 \\
\cline { 2 - 3 } & Fac. de Económicas y Empresariales & 9.1 \\
\cline { 2 - 3 } & Fac. de Derecho & 6.8 \\
\cline { 2 - 3 } & Fac. de Turismo y Finanzas & 3.8 \\
\hline Científico-Técnica & Escuela Técnica Superior de Ingeniería & 2.4 \\
\cline { 2 - 3 } & Escuela Técnica Superior de Ingeniería de la Edificación & 4.6 \\
\hline
\end{tabular}

Tabla 1. Facultades participantes según ramas de conocimiento de la Universidad de Sevilla

(España). (Fuente de elaboración propia).

\section{Procedimiento}

Se ha adaptado el Cuestionario de Intereses Curriculares del Alumnado Universitario (CICAU) de Barroso (2001). Una vez adaptado y actualizado, se ha desarrollado la validez de constructo, fiabilidad y consistencia interna del nuevo cuestionario.

Para la validez de constructo se realizó una revisión bibliográfica exhaustiva sobre el tema objeto de estudio y consulta a 110 estudiantes sobre el contenido del cuestionario. Una vez verificadas las modificaciones planteadas por los estudiantes, para garantizar la validez de constructo fue hallado el peso de cada una de las dimensiones e ítems a través de un Análisis Factorial Confirmatorio (AFE). De manera que los ítems cuyo peso fue valorado por debajo de .70 se eliminaron del cuestionario. Además de considerar que cada dimensión establecida estaba bien configurada si había tenido, al menos, tres ítems con pesos grandes (Beavers et al., 2013). El valor de coeficiente alfa de Cronbach para ambos cuestionarios indicó una alta consistencia interna. Para el Cuestionario de Intereses Curriculares del Alumnado Universitario (CICAU) fue de .72 y la adaptación del instrumento que hemos empleado en nuestra 
investigación Cuestionario sobre Expectativas del Alumnado Universitario (CEAU) es de .94 .

\section{Estructura del Cuestionario}

El total de 145 ítems que conformaron la adaptación del Cuestionario sobre Expectativas del Alumnado Universitario (CEAU) han sido agrupados en cuatro factores/dimensiones: personal, universidad, curricular y docencia. Las pruebas realizadas permiten considerar que la matriz de correlaciones resulta idónea para la aplicación de un análisis factorial (medida de adecuación de muestreo de KaiserMeyer-Olkin $=.861 \mathrm{y} \mathrm{p}=.000$ ).

El cuestionario ha sido cumplimentado por 1082 estudiantes de la Universidad de Sevilla, de las ramas del conocimiento de humanidades, bio-sanitaria, económicajurídica y científico-técnica.

\section{Análisis de datos}

Para completar la descripción de las percepciones del alumnado universitario sobre sus expectativas, hemos realizado un análisis diferencial de las dimensiones personal, universidad, curricular y docencia, analizadas en función de las ramas del conocimiento y titulación en la que cursan sus estudios. Hemos comenzado por calcular para cada estudiante la puntuación promedio alcanzada en el conjunto de ítems agrupados en una misma dimensión y, determinar posteriormente, la media de tales puntuaciones en el conjunto de alumnado de una misma rama de conocimiento. A través del análisis de la varianza se han contrastado los valores medios en cada rama, con el fin de determinar si las diferencias observadas resultan estadísticamente significativas. Con posterioridad a la realización del análisis de la varianza, al confirmarse la existencia de diferencias significativas ha sido conveniente investigar qué medias son distintas. Para ello, se han empleado diversas técnicas cuyo objeto ha sido identificar qué tratamientos son estadísticamente diferentes y en cuánto oscila el valor de esas diferencias. Por un lado, el procedimiento general de Scheffé, basado en la construcción de intervalos de confianza simultáneos para todas las posibles diferencias de medias y que permite su extensión a comparaciones más generales denominadas contrastes; y de otro lado, los contrastes de rangos múltiples basados en la distribución del rango estudentizado de Tukey.

Los resultados de estos análisis se presentan en función de las cuatro dimensiones identificadas al explorar la estructura del Cuestionario sobre Expectativas del Alumnado Universitario (CEAU).

\section{a) Dimensión Personal}

Las expectativas que presentan los estudiantes encuestados, en relación a la dimensión personal, son valoradas de forma similar en las diferentes ramas del conocimiento. Como se muestra en la Figura 1, las puntuaciones medias en la dimensión personal son prácticamente las mismas para las ramas bio-sanitaria, 
económico-jurídica y científico-técnica, donde oscilan entre los valores 3,26 y 3,29. Ligeramente superior es la media para la rama de humanidades alcanzando una puntuación de 3,43.

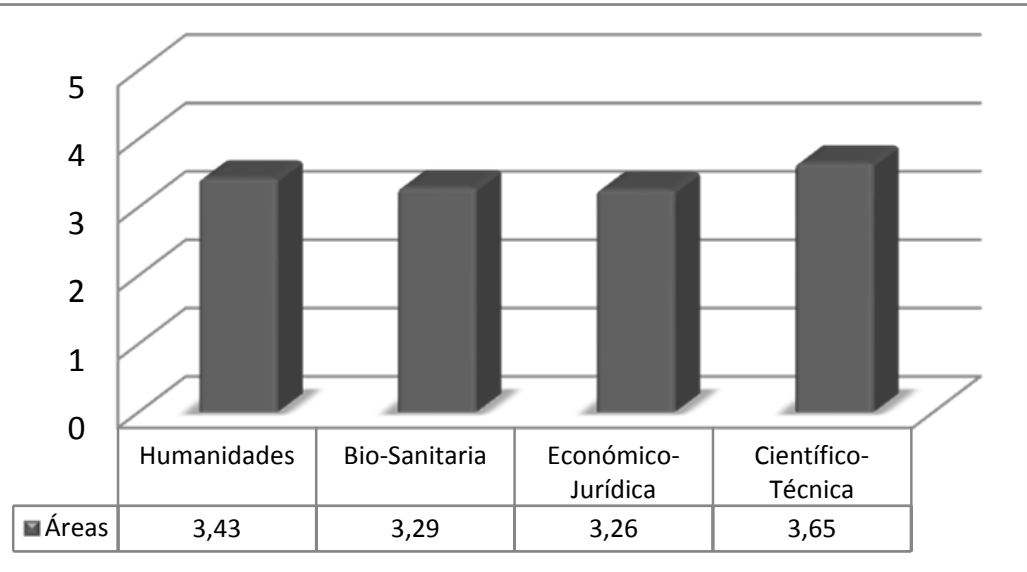

Figura 1. Media Dimensión Personal en función de la rama del conocimiento.

(Fuente de elaboración propia).

Esa ligera diferencia de la rama de humanidades respecto de las ramas bio-sanitaria y económico-jurídica es estadísticamente significativa al aplicar el análisis de la varianza para la comparación de medias (asumiendo varianzas iguales y prueba Scheffé). Como resultado de dichas pruebas obtenemos un valor de $F=7,631$ y $p=, 000$.

Si se observa la Figura 2, se pueden comparar las medias alcanzadas por cada uno de los ítems en cuestión, en función de la rama del conocimiento. Tal y como se aprecia, para el ítem 2 "en qué medida se han cumplido las expectativas que tenías sobre la Universidad", los estudiantes procedentes de la rama económico-jurídica manifiestan mayores expectativas que los estudiantes de la rama de humanidades. Por otro lado, si se comparan las ramas de humanidades y bio-sanitaria, se puede corroborar cómo los primeros manifiestan mayores expectativas frente a los segundos.

Otro ítem que alude a las expectativas pero, en este caso, a las expectativas con respecto al profesorado universitario (ítem 4), de nuevo, los estudiantes procedentes de la rama económico-jurídica manifiesta un mayor valor de media. Al igual que para el ítem anterior, si se comparan en este caso, los valores de respuesta entre las ramas de humanidades y bio-sanitaria, las diferencias son a favor de la rama de humanidades.

Con respecto al ítem "grado de interés por las actividades culturales" (ítem 9.1), cabe destacar que la rama de humanidades es la que tiene un valor de media mayor y la diferencia es significativa para esta rama.

En relación con las "campañas de sensibilización sobre determinados temas sociales" (ítem 9.3) la rama bio-sanitaria muestra valores más altos frente a la de humanidades; para "congresos y jornadas" (ítem 9.4), de nuevo, los estudiantes de biosanitaria muestra mayor interés frente a los de la rama de humanidades; y, por último, 
atendiendo a las "conferencias" (ítem 9.6), los de bio-sanitaria muestra mayor interés por las conferencias que los de humanidades.

Para los ítems sobre las expectativas referidas a "si creen que la enseñanza universitaria necesita cambiar", "en qué medida se vive en la Universidad un ambiente científico de investigación, búsqueda y actualización del conocimiento" y "si se vive en la Universidad un ambiente propicio a las relaciones humanas", las diferencias de medias son a favor de la rama de humanidades tras aplicar el análisis diferencial (ANOVA).

En lo que todos los estudiantes de la Universidad de Sevilla han coincidido es que la encuesta para valorar a los profesores no es un indicador fiable para la calidad de la docencia.

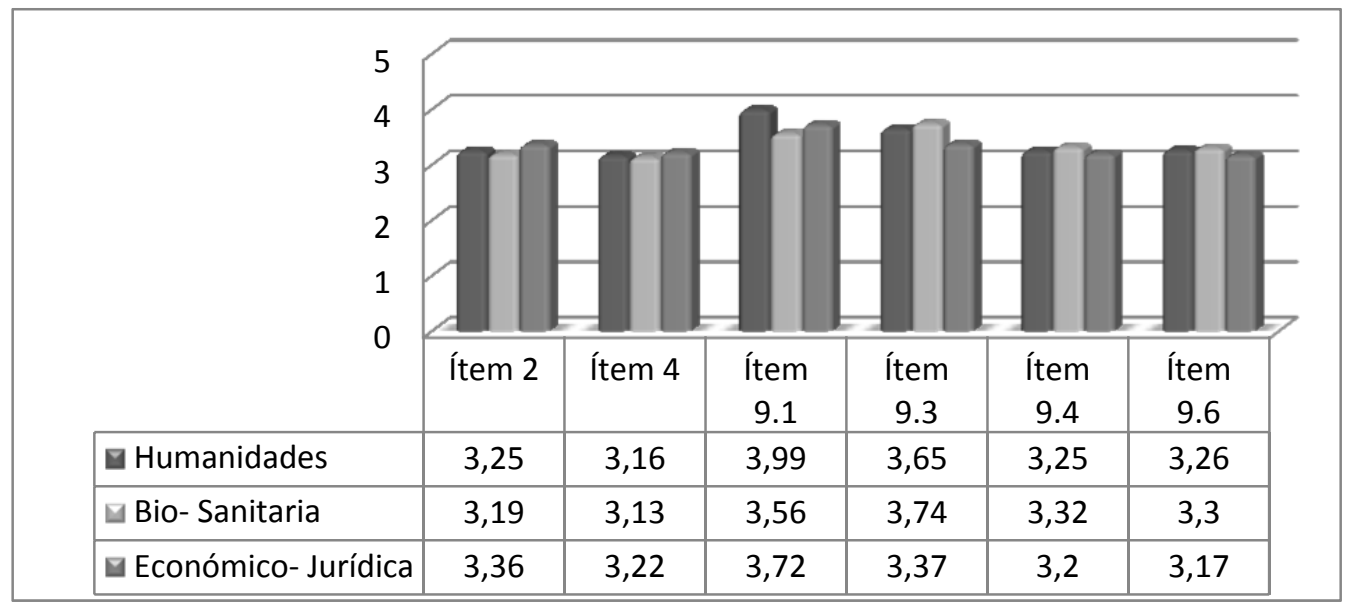

Figura 2. Comparación de medias para los ítems referidos a la Dimensión Personal.

(Fuente de elaboración propia).

\section{b) Dimensión Universidad}

En relación a la segunda dimensión se han agrupado los ítems que nos aportan información relativa a las expectativas de los estudiantes sobre los tipos de estudios, las prácticas y salidas profesionales, la participación en órganos de gobierno de la Universidad y las dependencias e instalaciones propias de la facultad, las puntuaciones de media alcanzadas son muy similares para las cuatro ramas del conocimiento (ver Figura 3).

Las diferencias observadas entre dichas ramas, tras aplicar el análisis de la varianza para la comparación de medias (asumiendo varianzas iguales y prueba Scheffé), no han resultado estadísticamente significativas, siendo los valores de $\mathrm{F}=0,949$ y $\mathrm{p}=, 417$. 


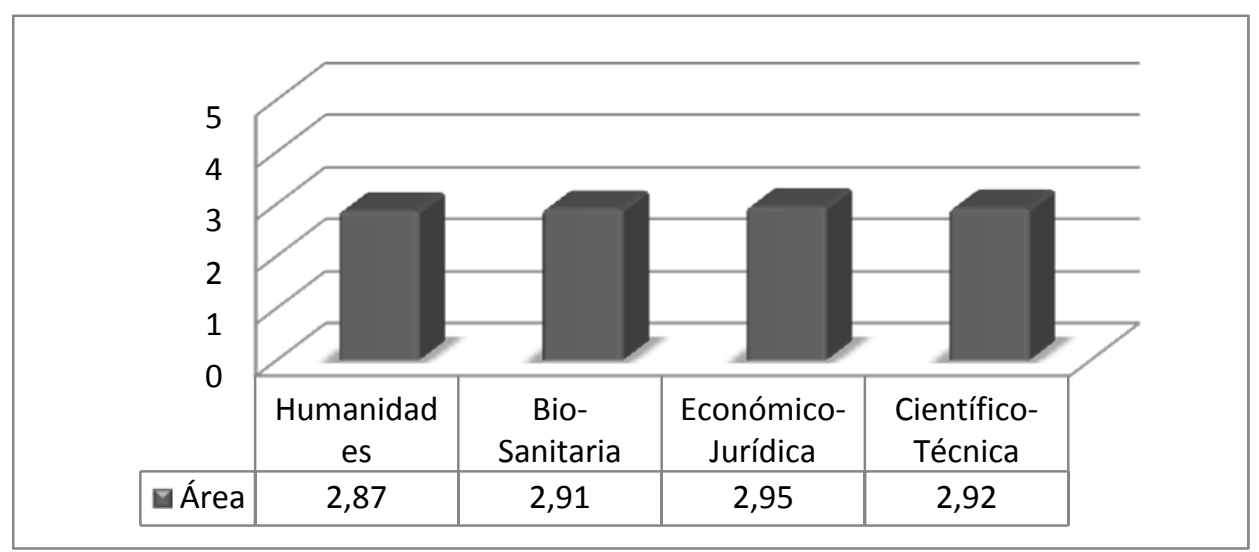

Figura 3. Media Dimensión Universidad en función de la rama del conocimiento. (Fuente de elaboración propia).

\section{c) Dimensión Curricular}

Los resultados arrojados para esta dimensión, muestran puntuaciones similares para las ramas del conocimiento bio-sanitaria, económico-jurídica y científico-técnica, como se puede observar en la Figura 4. De nuevo, la puntuación de la rama de humanidades es ligeramente superior a la media registrada por las demás ramas.

El análisis de varianza para las medias en esta dimensión (aplicando la prueba de Tukey) nos revela que existen diferencias significativas entre las medias de la rama de humanidades y la rama bio-sanitaria $(\mathrm{F}=2,851 \mathrm{y} \mathrm{p}=, 036)$.

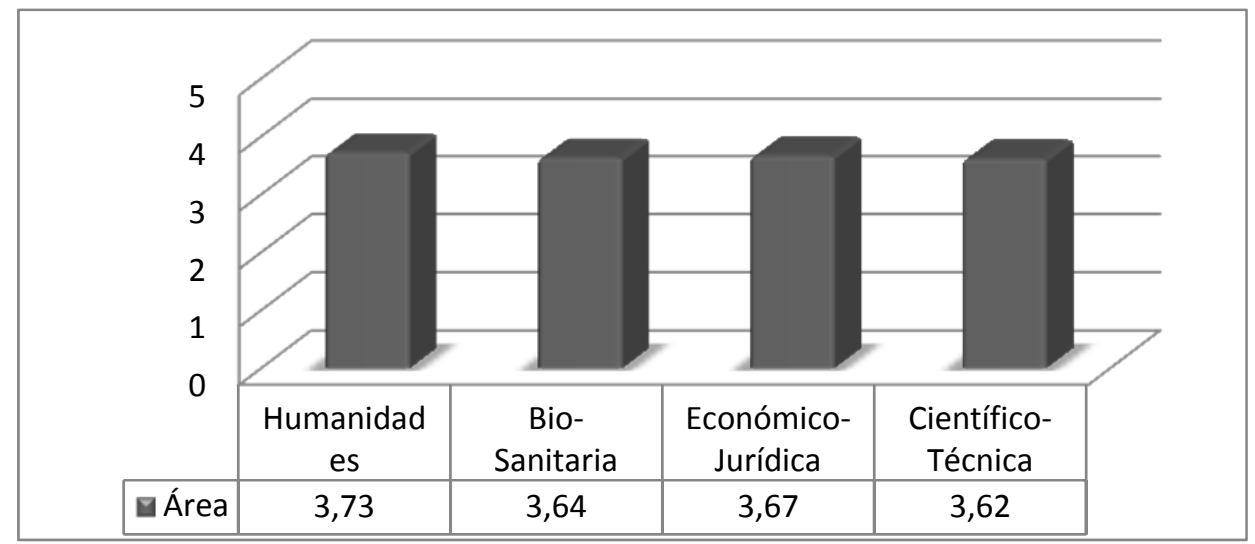

Figura 4. Medias Dimensión Curricular en función de la rama del conocimiento.

(Fuente de elaboración propia).

Si se atiende a las Figuras 5, 6, 7 y 8, se puede observar la comparación de medias en función de las ramas implicadas. Se ha procedido a analizar con mayor detalle aquellos ítems para los cuales el valor de media es superior en la rama bio-sanitaria, ya 
que según los datos arrojados a través de ANOVA, las diferencias significativas son para la rama de humanidades frente a la rama bio-sanitaria (obsérvese valor de medias en Figura 4).

Con respecto a los ítems que hacen referencia a los objetivos (ítem 11 y 12), una buena parte de ellos (seis concretamente) obtienen valores de media superiores en la rama bio-sanitaria frente a la rama de humanidades. Los ítems en cuestión hacen alusión al interés de los estudiantes porque los objetivos sean elaborados por el profesorado (ítem 11.1), por un grupo de personas relevantes (ítem 11.3), por la Universidad y las empresas demandantes de empleo (ítem 11.4), por las verdaderas necesidades del mercado de trabajo (ítem 11.5), por los departamentos (ítem 11.6) y, que a través de los mismos se consigan los conocimientos teóricos de las asignatura (ítem 12.6).

A pesar que para la dimensión curricular, según la aplicación de ANOVA y la prueba de Tukey, existen diferencias significativas en las ramas de humanidades y biosanitaria (a favor de la primera), para los ítems mencionados con anterioridad, el valor de media es mayor para la rama bio-sanitaria.

En relación con los ítems referidos a los contenidos (ítems 13 y 14), aspectos que caben destacar son el ítem 13.2, "interés por la existencia de contenidos dirigidos a desarrollar la ética profesional", el ítem 14.1 y 14.3, "interés por que los temas de las asignaturas tengan en cuenta su valor científico y su utilidad profesional" respectivamente. Para estos tres ítems, a diferencia que para el resto, los valores de media más altos son para la rama bio-sanitaria sobre la de humanidades. Los ítems dirigidos a fomentar la iniciativa económica, formación en valores, formación política y formación intelectual tienen puntuaciones superiores en la rama de humanidades.

Para los ítems relativos a las actividades (ítems 15 y 16), únicamente cuatro de ellos obtienen medias más elevadas en la rama bio-sanitaria. Estos ítems son el 15.2, las actividades pongan en práctica las teorías estudiadas, el ítem 16.1, 16.6 y 16.7, que aluden al "desarrollo de actividades con profesionales ajenos a la facultad", al "desarrollo de actividades individuales" y al "desarrollo de actividades en laboratorios", respectivamente. Si se atiende a éste último ítem, "desarrollo de actividades en laboratorios", el valor de media alcanzado por la rama bio-sanitaria es mucho mayor que el alcanzado por la rama de humanidades (obsérvese Figura 6). El resto de ítems "motiven a los estudiantes, potencien la participación creativa, investiguen profesores y alumnos, grupos de no más de tres personas, colaboren con empresas" las diferencias más significativas las encontramos en la rama de humanidades. 


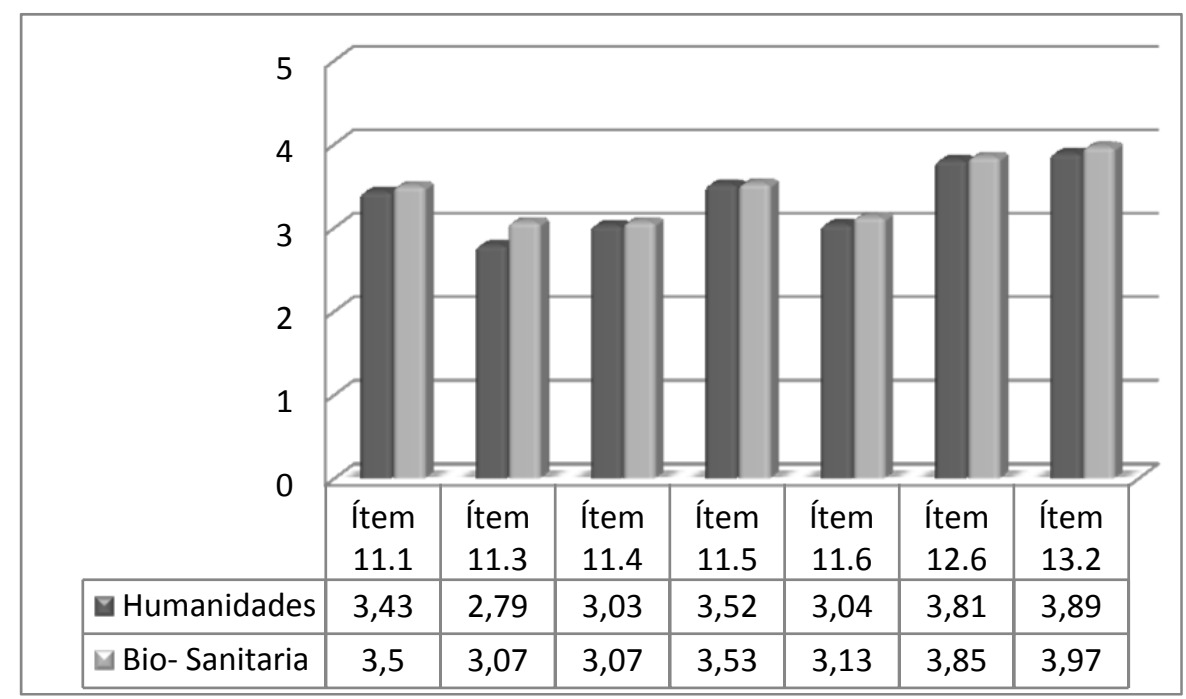

Figura 5. Comparación de medias para los ítems referidos a la Dimensión Curricular (del ítem 11 al 13). (Fuente de elaboración propia).

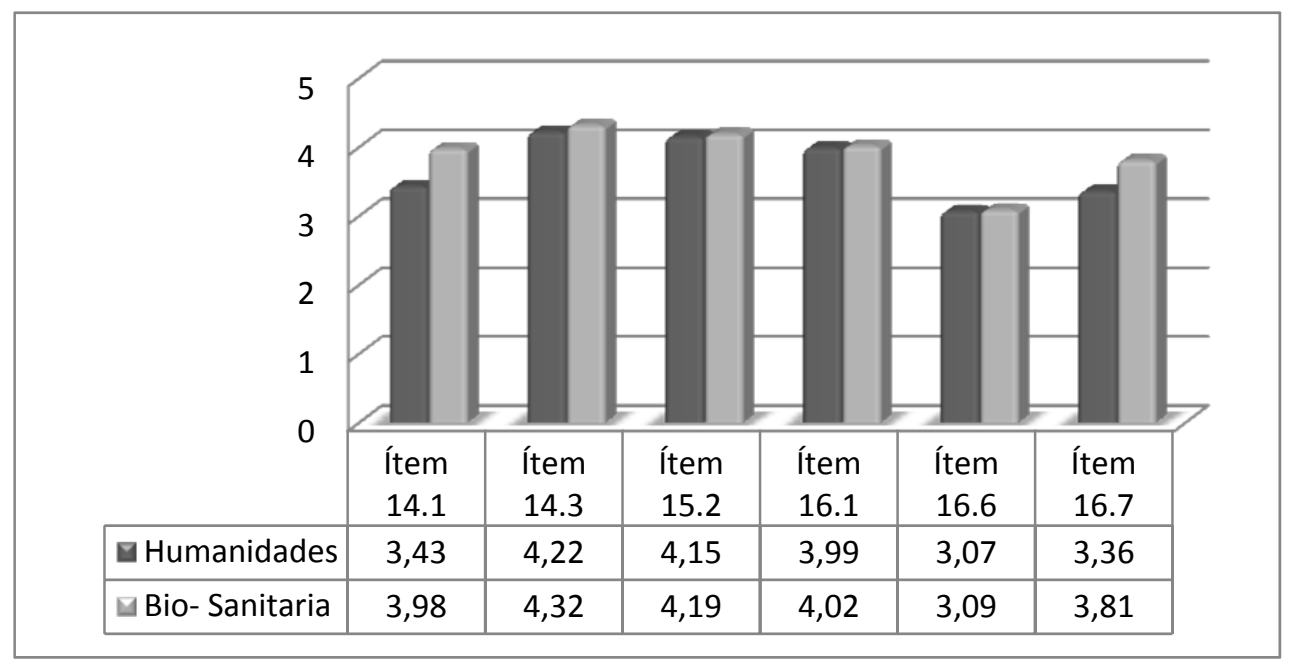

Figura 6. Comparación de medias para los ítems referidos a la Dimensión Curricular (del ítem 14 al 16). (Fuente de elaboración propia).

En relación con las estrategias de enseñanza (ítems 17 y 18), únicamente para el ítem 18.2, con referencia a que el profesorado utilice la lección magistral como norma, el valor de media es superior en la rama bio-sanitaria. Para el resto de ítems, "discutir los temas, demostración del profesor replicada por los estudiantes, partir del trabajo previo del alumno y aclarar dudas, dar a la asignatura un enfoque crítico, 
mentorización de alumnos de cursos superiores", las diferencias son para la rama de humanidades. En este caso, las diferencias entre las ramas de humanidades y biosanitarias con respecto a las estrategias de enseñanza son bastante notorias.

Para los ítems relacionados con los medios utilizados en la enseñanza (ítem 19), las diferencias más significativas se encuentran en la rama de humanidades como se aprecia en los resultados obtenidos a través de la aplicación de la prueba ANOVA. Los estudiantes de la rama de humanidades muestran mayor interés por el uso de medios de enseñanza frente a los de la rama bio-sanitaria. Las diferencias más representativas entre dichas ramas son para los ítems 19.2, "uso de pizarra digital", 19.4, "uso del proyector de transparencias" y 19.1, "uso de la pizarra de encerado", ordenadas en función de criterio descendente.

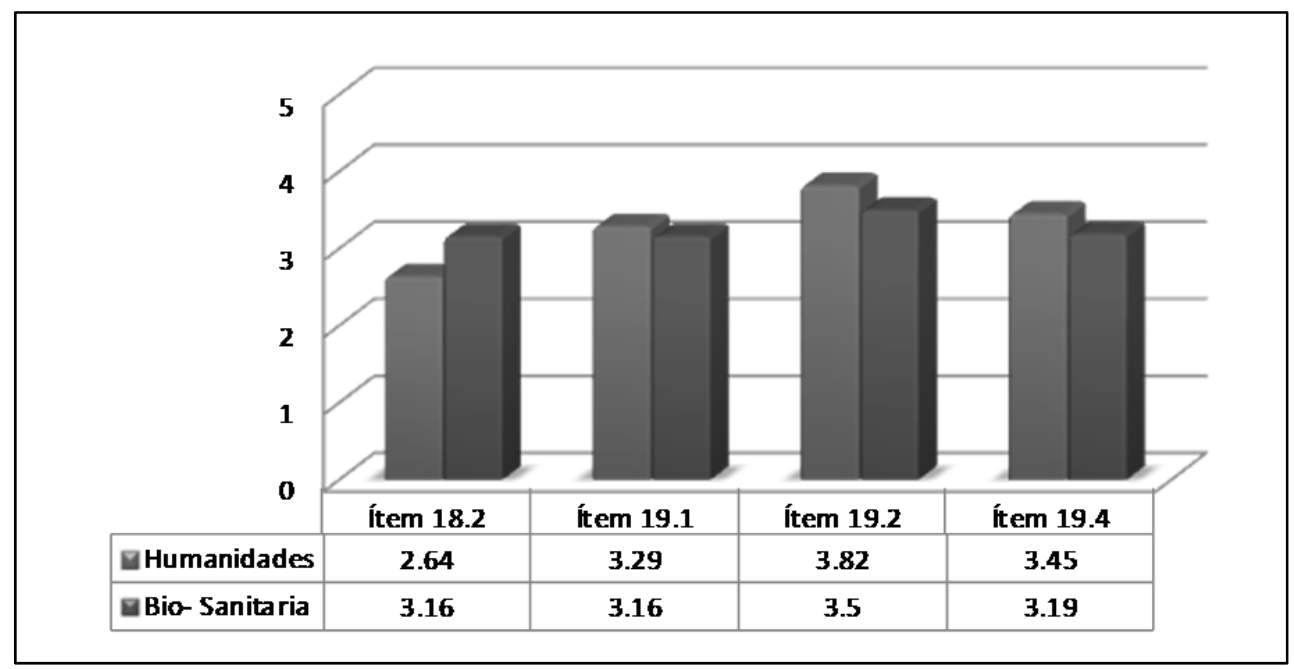

Figura 7. Comparación de medias para los ítems referidos a la Dimensión Curricular (del ítem 17 al 19). (Fuente de elaboración propia).

Por último, en relación con los ítems referidos a la evaluación (ítems 20 y 25), a diferencia que en casos anteriores, la mayoría de ítems (diez de catorce) obtienen valores de media superiores para la rama bio-sanitaria. Estos ítems son 20.2, "interés por que el profesorado califique mediante exámenes con pruebas objetivas", ítem 20.3, "interés por que el profesorado evalúe los conocimientos mediante el examen y los trabajos para subir nota", ítem 20.4, "interés por realizar un examen escrito al acabar cada tema", ítem 20.7, "interés por realizar exámenes con apuntes", ítem 25.1, "interés por que el profesorado califique ateniéndose a unas normas preestablecidas", ítem 25.2, "interés por que el profesorado corrija los exámenes y pruebas con rapidez", ítem 25.3, "interés por que el profesorado juzgue a los estudiantes por sus trabajos y no por otros aspectos", ítem 25.4, "interés por que el profesorado no admita favores personales del alumnado", ítem 25.5, "interés por que el profesorado sea generoso en 
las calificaciones poniendo notas altas", y, por último, el ítem 25.7, "interés por que el profesorado le dé más valor a la parte teórica que a la práctica".

Como puede observarse, la evaluación, dentro de la dimensión curricular, es un aspecto al que los estudiantes de la rama bio-sanitaria otorgan especial interés.

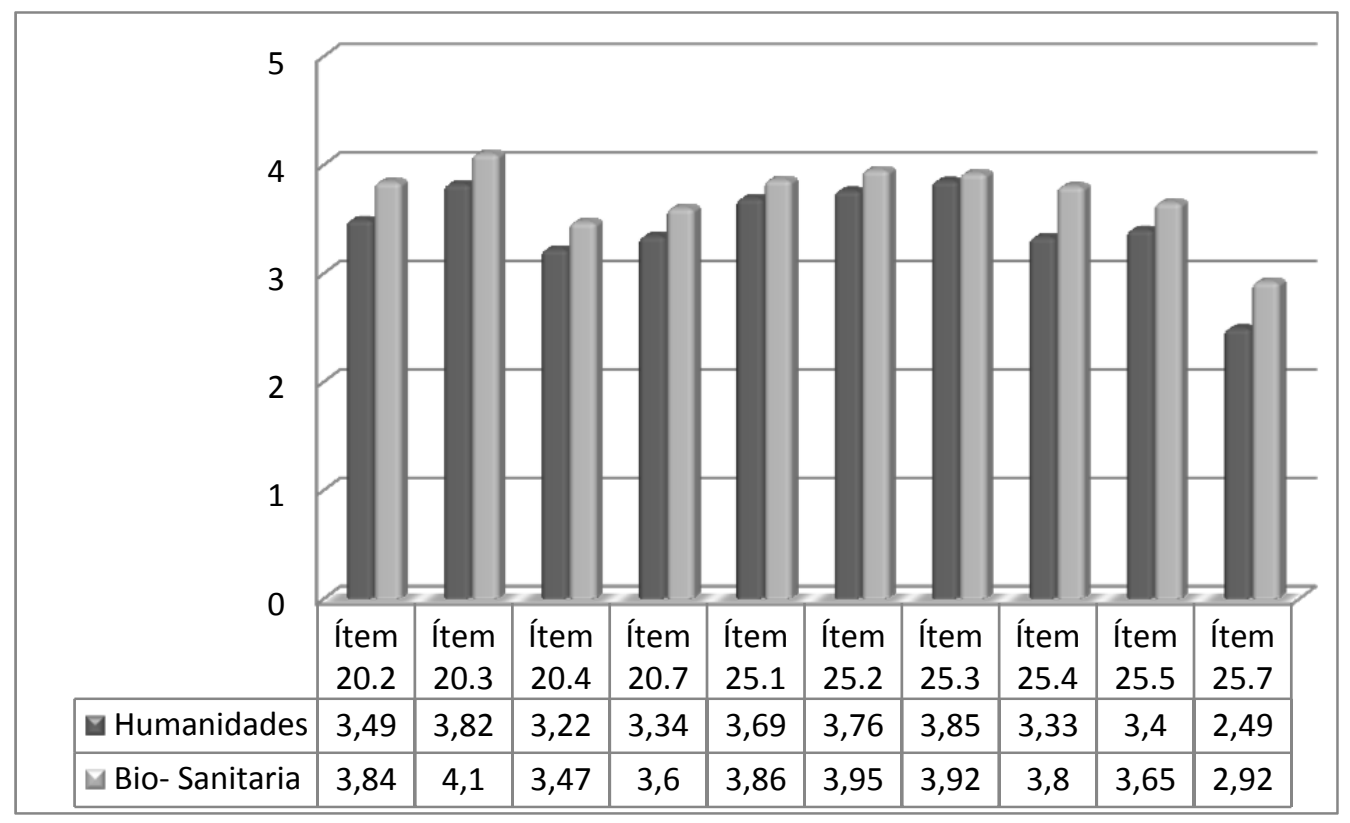

Figura 8. Comparación de medias para los ítems referidos a la Dimensión Curricular (del ítem 20 al 25). (Fuente: elaboración propia).

\section{d) Dimensión docencia}

En relación con las puntuaciones medias alcanzadas en esta dimensión, al igual que para dimensiones anteriores, en las ramas bio-sanitaria, económico-jurídica y científico-técnica son muy similares, oscilando entre 3,54 y 3,59. De nuevo, ligeramente superior es la media alcanzada para la rama de humanidades cuya puntuación alcanza el valor de 3,66 (Ver Figura 9). 


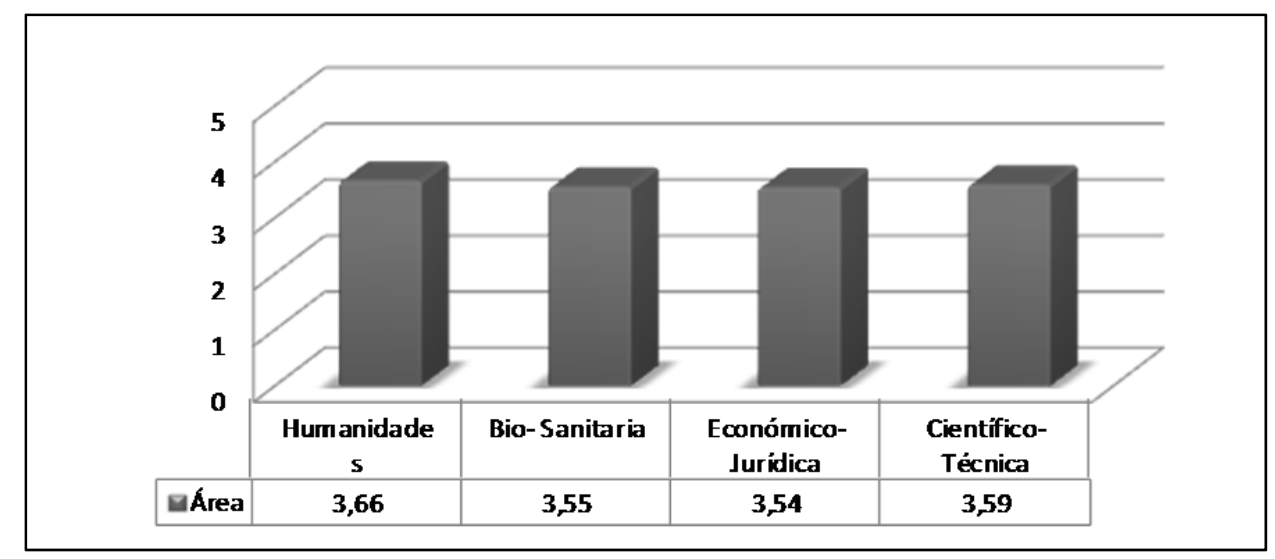

Figura 9. Medias Dimensión docente universitario en función de la rama del conocimiento. (Fuente: elaboración propia).

El análisis de la varianza y la aplicación de la prueba de Scheffé refleja que estas opiniones registradas difieren significativamente entre las ramas de humanidades y biosanitaria, y humanidades y económico-jurídica $(\mathrm{F}=4,034$ y $\mathrm{p}=, 007)$.

Si se observan las Figuras 10 y 11, se pueden comparar las medias alcanzadas por cada uno de los ítems que componen esta dimensión. A su vez, se pueden diferenciar los valores medios registrados en cada rama del conocimiento implicada en el resultado del análisis diferencial de varianzas (ANOVA). 


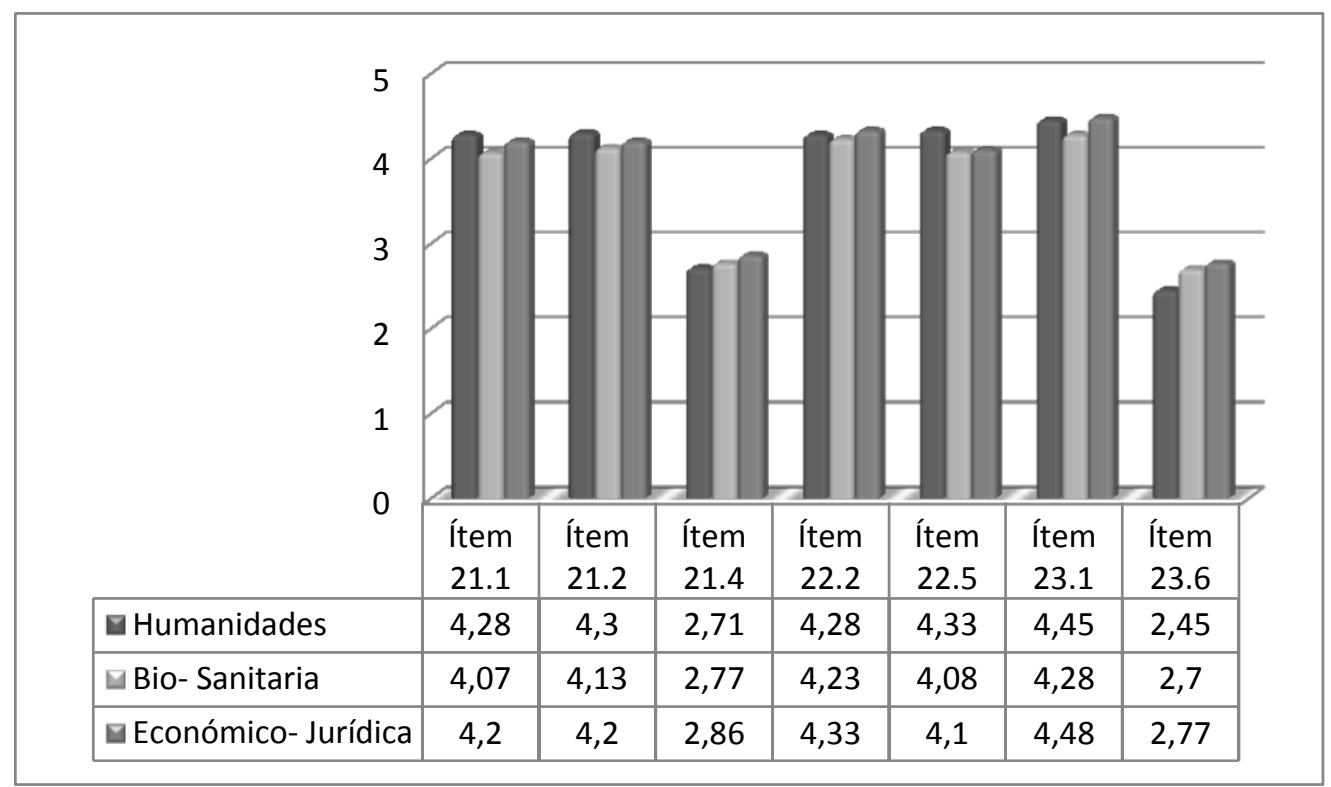

Figura 10. Comparación de medias para los ítems referidos a la Dimensión docente (del ítem 21 al 23). (Fuente: elaboración propia).

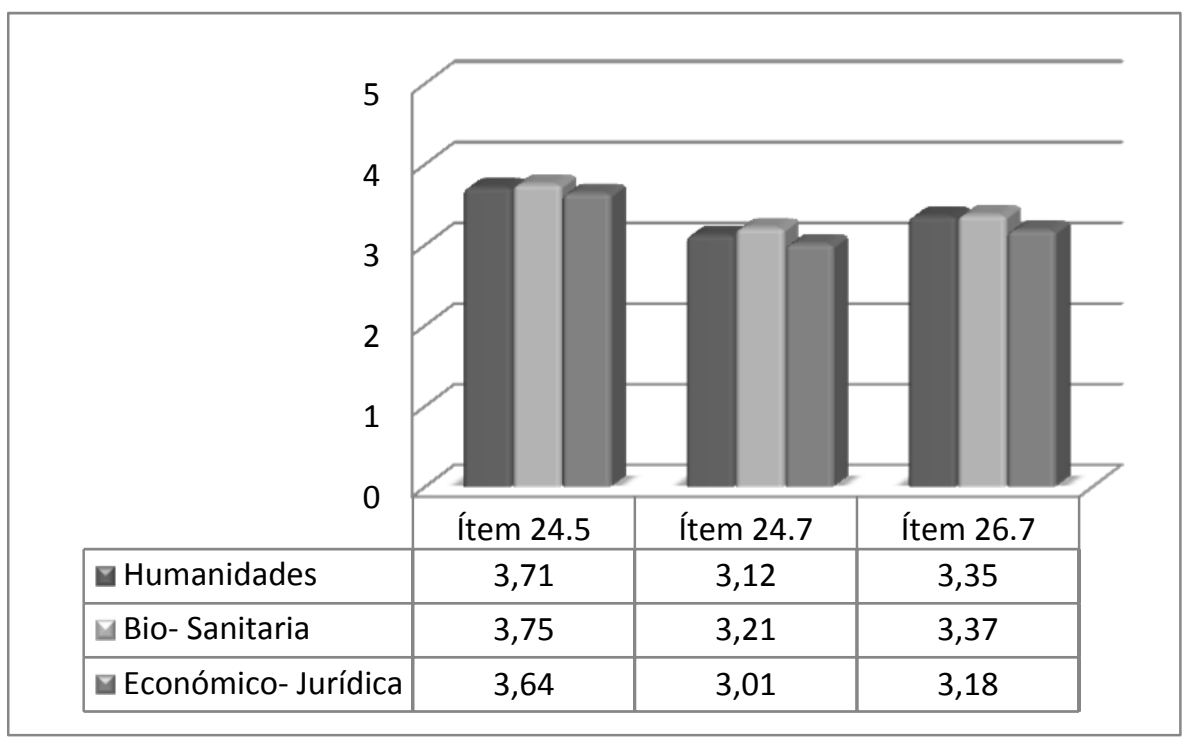

Figura 11. Comparación de medias para los ítems referidos a la Dimensión docente (del ítem 24 al 26). (Fuente: elaboración propia). 
Con respecto a los ítems sobre la personalidad del profesorado (ítem 21), en el que deben valorar su grado de interés porque "el profesor sea sincero, honrado, atento y tolerante, correcto en su forma de vestir, bondadoso, simpático y con sentido del humor". Los estudiantes de humanidades registran los valores de media más elevados para casi la totalidad de los ítems. El único ítem cuyo valor de media es inferior para humanidades es el ítem 21.4, que hace referencia al grado de interés por la corrección en la forma de vestir del profesorado.

Atendiendo a la actitud del profesorado con respecto a la enseñanza de su materia (ítem 22), en el que deben "valorar el interés y entusiasmo por la materia que imparte el profesor", "estar al día en la disciplina que enseña", "establecer relaciones con otras áreas de conocimiento", "tener una perspectiva crítica", "tener en cuenta los conocimientos previos de los estudiantes", "incluir en su programa una formación humana" y "demostrar entusiasmo". Para el ítem 22.2, "interés por que el profesorado esté al día de la disciplina que enseña", el valor medio máximo no lo registra la rama de humanidades sino la rama económico-jurídica. Y, por otro, el ítem sobre que el profesorado tenga en cuenta los conocimientos previos de los estudiantes (ítem 22.5) es más valorado por la rama de humanidades. Para el resto de ítems, los valores máximos registrados han sido para la rama de humanidades.

Los ítems referidos a la metodología utilizada por el profesorado (ítem 23), para dos de ellos, los valores de media son favorables a la rama del conocimiento económicojurídica en detrimento de la rama de humanidades. Estos son el ítem 23.1, "interés por que el profesorado motive a su alumnado para que se interese por la materia" y el ítem 23.6, "interés por que predomine la parte teórica sobre la práctica en las distintas asignaturas". Para este último ítem se puede comprobar como los estudiantes de la rama de humanidades muestran menor interés por el predominio de la teoría frente a la práctica al contrario que los de las ramas bio-sanitaria y económico-jurídica.

En la relación profesorado-alumnado (ítem 24), para la mayoría de los ítem los valores de media son favorables a la rama de humanidades respecto a las ramas biosanitaria y económico-jurídica. Dos casos constituyen la excepción en este aspecto. Para el ítem 24.5, "interés por que el profesorado realice investigaciones con la colaboración de los estudiantes" y el ítem 24.7, "interés porque el profesor sea amigo de sus estudiantes", los valores de media alcanzan sus valores máximos para la rama bio-sanitaria.

En última instancia y, en relación con la vida académica y comunitaria del profesorado (ítem 26), el único ítem para el que la media no es favorable a la rama de humanidades y sí a la rama bio-sanitaria es el 26.7, que hace referencia al "interés por que el profesorado pertenezca a un grupo de investigación". Para el resto de ítems, "publicar libros", "escribir artículos", "participar activamente en proyectos de investigación", "dar conferencias", "desempeñar cargos de responsabilidad" y "desarrollar un trabajo paralelo a su labor docente", los valores de media son superiores en la rama de humanidades. 


\section{Conclusiones}

Con esta investigación se ha pretendido analizar las expectativas que poseen los estudiantes de la Universidad de Sevilla, durante el curso académico 2012-2013, estableciendo un análisis diferencial en función de la rama del conocimiento en la que han cursado sus estudios.

Atendiendo a las expectativas de los estudiantes encuestados sobre la Dimensión Personal, son valoradas de forma similar en las cuatro ramas del conocimiento. Aunque sí se destaca que los estudiantes de la rama económico-jurídica son los que manifiestan mayores expectativas sobre su Universidad y sobre el profesorado. Pero al preguntar por las expectativas en las actividades que organiza la Universidad, son los estudiantes de bio-sanitaria los que destacan por su interés en campañas de sensibilización sobre temas sociales, congresos, jornadas y conferencias. En cambio, para las expectativas referidas a "si creen que la enseñanza universitaria necesita cambiar", "en qué medida se vive en la Universidad un ambiente científico de investigación, búsqueda y actualización del conocimiento" y "si se vive en la Universidad un ambiente propicio a las relaciones humanas" son los de humanidades lo que presentan mayores puntuaciones.

En lo que todos los estudiantes de la Universidad de Sevilla han coincidido es que la encuesta para valorar a los profesores no es un indicador fiable para la calidad de la docencia. Año tras año se realizan evaluaciones al profesorado pero no se revisan las preguntas realizadas a los estudiantes para poder mejorar la docencia en la Universidad. Este hecho puede ser debido a la preocupación focalizada en las acreditaciones a ANECA y no en un análisis en profundidad de la institución y su profesorado.

Para la Dimensión Universidad, los tipos de estudios, prácticas y salidas profesionales, la participación en Órganos de Gobierno de la Universidad y las dependencias e instalaciones propias de la Facultad, las puntuaciones de media alcanzadas son muy similares para las cuatro ramas del conocimiento; casi todos los estudiantes encuestados reclaman una formación que dé respuesta a las necesidades del mercado de trabajo. En cuanto a los Órganos de Gobierno, ni participan ni están interesados en participar. En la mayoría de los casos no suelen participar por falta de información o por pasotismo. Estudios como los de Salaburu (2007); Gonzalez (2007), Michavila y Parejo (2008) y Jover Olmeda, López Martín y Quiroga Uceda (2011) demuestran la escasa participación que los estudiantes españoles tienen en la vida universitaria, en comparación con lo que ocurre en otros contextos como el anglosajón o el nórdico. Estamos convencidas que el principal elemento para participar es el apoyo de la Universidad con programas de dinamización, mayor presupuesto, fomentar la representación estudiantil, porcentaje de estudiantes en órganos colegiados, etc. La Universidad de Sevilla debe plantearse si tiene una difusión suficiente y efectiva y si la información llega al sector estudiantil. Estos indicadores tienen relación con la participación de los estudiantes de la Universidad y sin duda, esta actitud ha tenido influencia en las expectativas de los estudiantes. 
Para la Dimensión Curricular los ítems sobre objetivos y contenidos obtienen valores de media favorables a la rama bio-sanitaria con respecto a la de humanidades. Los estudiantes de ésta rama consideran que los objetivos deben ser elaborados no sólo por los profesores, sino por personas relevantes, por la Universidad, empresas demandantes de empleo y por las necesidades del mercado de trabajo. Un aspecto muy interesante es que, los estudiantes de la rama bio-sanitaria, consideran que los contenidos deben ir dirigidos a desarrollar la ética profesional. Esto es debido a que el código deontológico es una práctica muy acusada, que regula y guía la actividad profesional de esta rama de conocimiento por encima de las otras ramas. En relación a las actividades obtienen medias favorables la rama bio-sanitaria; específicamente, las actividades que pongan en práctica las teorías estudiadas (Barroso, 2009), que aludan al desarrollo de actividades con profesionales ajenos a la Facultad, al desarrollo de actividades individuales y al desarrollo de actividades en laboratorio. Atendiendo a las estrategias de enseñanza los valores de media son superiores para la rama de humanidades (Barroso, 2009); dichos estudiantes muestran mayor interés por la utilización de métodos enseñanza más novedosos frente a los de la rama bio-sanitaria. De este modo, los estudiantes de la rama bio-sanitaria prefieren la lección magistral y los de humanidades y económico-jurídica prefieren discutir los temas, partir de su trabajo previo, aclarar dudas, permitir un enfoque de la ciencia más crítico y la mentorización con compañeros de cursos superiores. Para los ítems relacionados con los medios utilizados en la enseñanza, destaca los de humanidades, mostrando mayor interés por el uso de medios de enseñanza más innovadores frente a los de biosanitaria. Por último, en la evaluación la mayoría de ítems obtienen valores de media superiores para la rama bio-sanitaria en detrimento de la rama de humanidades. Este elemento curricular es muy valorado por los estudiantes de bio-sanitaria aunque consideren que los exámenes son la mejor forma de evaluar y que el profesorado debe calificar ateniéndose a unas normas preestablecidas. Los estudiantes de humanidades, en cambio, argumentan que las modalidades clásicas de calificación no discriminan sobre sus capacidades, por este motivo, es imprescindible ir más allá de la transmisión de conceptos y la repetición de informaciones para poder adquirir las competencias, habilidades y destrezas propias de su rama de conocimiento.

Por último, la Dimensión Docencia, es ligeramente superior la media alcanzada para los de humanidades. Con respecto a la personalidad del profesorado, los estudiantes de humanidades muestran mayor interés por las características de la personalidad del profesorado que los de las demás ramas; valorando principalmente la atención, tolerancia, honradez y sinceridad. Atendiendo a la actitud del profesorado con respecto a la enseñanza de su materia, el valor de media máximo lo registra la rama económico-jurídica. Y, por otro, el ítem más favorable de la rama de humanidades hace referencia al interés porque el profesorado tenga en cuenta los conocimientos previos de los estudiantes. En relación a los ítems referidos a la metodología utilizada por el profesorado, los estudiantes de humanidades muestran menor interés por el predominio de la teoría frente a la práctica y los de económicojurídica prefieren que los profesores motiven a los estudiantes y predomine la teoría sobre la práctica en algunas asignaturas. Con respecto a la relación profesoradoalumnado y, la vida académica y comunitaria del profesorado, los valores de media 
son favorables a la rama de humanidades respecto a la rama bio-sanitaria y económicojurídica. Los aspectos que más valoran los estudiantes universitarios son la participación activa del profesorado en proyectos de investigación, impartición de conferencias, la pertenencia a un grupo de investigación y la redacción de artículos para periódicos y revistas. En el caso de la rama bio-sanitaria plantean el interés por que el profesorado realice investigaciones en colaboración con los alumnos y que puedan ser amigos de los profesores.

La mayoría de los estudiantes tienen mayores expectativas en una buena formación que en una buena dedicación investigadora de sus profesores, por tanto, esperan y reclaman una dedicación docente de calidad, valorando ambas igualmente pero con la conciencia de que una alta calidad docente les influye más directamente que una alta calidad investigadora.

Los resultados de esta investigación avalan que las características de un buen docente deben situarse en dos vías: cómo es (vía relacional) y cómo hace su trabajo, labor docente, relaciones con alumnos y otras actividades que debe desarrollar (vía técnica). Para los estudiantes de humanidades el ámbito relacional es más importante que el técnico en un profesor y para los estudiantes de las ramas de bio-sanitaria y económico-jurídica el ámbito técnico posee mayor importancia.

Para finalizar, comentar que la escasa participación del profesorado de la rama científico-técnica ha supuesto una gran limitación, por no poder conocer en profundidad las expectativas de los estudiantes de esta rama del conocimiento, que ha escapado al control de esta investigación.

En futuros trabajos se completarán estos resultados con las valoraciones de Universidades españolas, ya que la aspiración a una permanente mejora en la formación universitaria requiere considerar las valoraciones y el nivel de satisfacción logrado entre los usuarios de las instituciones de educación superior.

\section{Referencias bibliográficas}

AGUADED, J. I. Y LÓPEZ, E. (2009). La evaluación de la calidad didáctica de los cursos universitarios en red: diseño e implementación de un instrumento, Enseñanza \& Teaching, 27(1), 95-114.

ALONSO, J. (1999). Comportamiento del consumidor. Madrid: ESIC.

APODACA, P. Y GRAD, H. (2002). Análisis dimensional de las opiniones de los alumnos universitarios sobre sus profesores. Revista de Investigación Educativa, 20 (2), 385-409.

BENEDICTO, J. Y MORÁN, M. L. (1999). Aprendiendo a ser ciudadanos. Experiencias sociales y construcción de la ciudadanía entre los jóvenes, Madrid: Injuve, Ministerio de Trabajo y Asuntos Sociales.

BARROSO, P. (2001). Intereses Curriculares de los Alumnos Universitarios. Tesis Doctoral inédita: Universidad de Sevilla. 
BARROSO, P. (2009). Investigación Educativa: enseñar en la Universidad. Sevilla: Edición Digital@tres, S.L.L.

BEAVERS, A. S., LOUNSBURY, J. W. RICHARDS, J. K., HUCK, S. W., SKOLITS, G. J. Y ESQUIVEL, S. L. (2013). Practical considerations for using exploratory factor analysis in educational research. Practical assessment, research \& evaluation, 18 ( 6), Recuperado de : http://pareonline.net/pdf/v18n6.pdf.

BELTRÁN, J. A. (2000). Intervención psicopedagógica y currículo escolar, Madrid: Pirámide.

COSACOV, E. (2007). Diccionario de Términos Técnicos de Psicología, 3ra Ed., Córdoba: Brujas.

DE LA ORDEN, A. (2009). Evaluación y calidad: análisis de un modelo, Estudios Sobre Educación, 16, 17-36.

DOWNIE, N. M. Y HEATH, R. W. (1983). Métodos Estadísticos Aplicados. Madrid: Del Castillo.

ELIOPHOTOU, M. (2003). Student Involment in University Governance: a Need for Negotiated Educational Aims? Tertiary Education and Management, 9, 233-246.

ENTWISTLE, N. Y TAIT, H. (1990). Approaches to learning, evaluations of teaching, and preferences for contrasting academic environments Higer Education, 19, 169194.

FERNÁNDEZ PÉREZ, M. (1989). Así enseña nuestra universidad. Hacia la construcción crítica de una didáctica universitaria. Salamanca: Hupagraphis.

FUENTES, E. J. Y GONZÁLEZ, M. (1997). El profesorado universitario: reflexiones en torno a su formación y a su desarrollo profesional. Adaxe, 13, 85-101.

GARCÍA GARDUÑO, J. M. Y MEDÉCIGO SHEJ, A. (2014). Los criterios que emplean los estudiantes universitarios para evaluar la in-eficacia docente de sus profesores. Perfiles educativos, 36 (143), 124-139. Recuperado de http://www.revistas.unam.mx/index.php/perfiles/article/viewFile/44026/39835

GIMENO, J. (2008). Educar por competencias. Que hay de nuevo?. Madrid: Morata.

GONZÁLEZ, I. (Coord.) (2007). Participació, politica i joves. Una aproximació a les pràctiques polítiques, la participació social i l'afecció política de la joventut catalana. Barcelona: Secretaria General de Joventut. Generalitat de Catalunya.

IBÁÑEZ, J. A. (1990). Dimensiones de la competencia profesional del profesor de Universidad, Revista Española de Pedagogía, 186, 239-255.

JOVER OLMEDA, G., LÓPEZ MARTÍN, E. Y QUIROGA UCEDA, P. (2011). La universidad como espacio cívico: valoración estudiantil de las modalidades de participación política universitaria. Revista de Educación, número extraordinario, 69-91. 
LEÓN, B. Y LATAS, C. (2005). Nuevas exigencias en el proceso de enseñanzaaprendizaje del profesor universitario en el contexto de la convergencia europea: la formación en técnicas de aprendizaje cooperativo Revista Interuniversitaria de Formación del Profesorado, 8 (6), 45-48.

LIZZIO, A. Y WILSON, K. (2009). Student Participation in University Governance: the Role Conceptions and Sense of Efficacy of Student Representatives on Departmental Comites. Studies in Higher Education, 34 (1), 69-84.

LÓPEZ, M.; CANNELLA, S. Y CIANCIMINO, E. (2010). Proyecto de evaluación mixta ALTA: la experiencia en un entorno mexicano de enseñanza a distancia. Revista Electrónica de Investigación Educativa, 12 (2), 1-6, Recuperado de http://redie.uabc.mx/contenido/vol12no2/contenido-lopezcannella.pdf

MARTÍNEZ GARCÍA, M. M. , GARCÍA DOMINGO, B. Y QUINTANAL DÍAZ, J. (2006). El perfil del profesor universitario de calidad desde la perspectiva del alumnado. Revista Educación, XX1 (9), 183-198.

MERHI, R. (2011). Expectativas del estudiantado en la universidad del nuevo milenio. Un proceso dinámico. La Cuestión Universitaria, 7, 23-31.

MICHAVILLA, F. Y PAREJO, J. L. (2008). Políticas de participación estudiantil en el Proceso de Bolonia. Revista de Educación, número extraordinario, 85-118.

PICHARDO, M. C., GARCÍA BERBÉN, A. B., DE LA FUENTE, J. Y JUSTICIA, F. (2007). El estudio de las expectativas en la universidad: análisis de trabajos empíricos y futuras líneas de investigación. Revista Electrónica de Investigación Educativa, 9 (1),1-16.

PORTO, M. (2006). La evaluación de los estudiantes universitarios vista por sus protagonistas. Educatio Siglo XXI, 24, 167-188.

RAMSDEN, P., MARTIN, E. Y BOWDEN, J. (1989). School environment and sixth form pupils' approaches to learning. British Journal of Educational Psychology, 59 (2), 129-142.

RICOY, Ma . C. Y FERNÁNDEZ-RODRÍGUEZ, J. (2013). La percepción que tienen los estudiantes universitarios sobre la evaluación: un estudio de caso. Educación $X X 1,16(2), 321-342$.

RODRÍGUEZ-AYÁN, M. N. (2005). La perspectiva estudiantil sobre el desempeño del profesor: un modelo de regresión logística ordinal. Revista Electrónica de Metodología Aplicada (REMA), 10 (1), 1-13.

SALABURU, P. (2007). La universidad en la encrucijada. Europa y EEUU, Madrid: Academia Europea de Ciencias y Artes.

SALVADOR, C. M. (2005). La percepción del cliente de los elementos determinantes de la calidad del servicio universitario: características del servicio y habilidades profesionales. Papeles del Psicólogo, 26 (90), 1-9. 
SÁNCHEZ, Ma . P. (2010). Técnicas docentes y sistemas de evaluación en Educación Superior. Madrid: Narcea.

SOLER, P.; PALLISERA, M.; PLANAS, A.; FULLANA, J. Y VILÁ, M. (2012). La participación de los estudiantes en la universidad: dificultades percibidas y propuestas de mejora. Revista de Educación, 358, 542-562.

STEINMANN, A., BOSCH, B. Y AIASSA, D. (2013). Motivación y expectativas de los estudiantes por aprender ciencias en la Universidad. Revista Mexicana de Investigación Educativa, 18 (57), 585-598 Recuperado de http://www.comie.org.mx/v1/revista/visualizador.php?articulo=ART57011\&criterio $=\mathrm{http}: / /$ www.comie.org.mx/documentos/rmie/v18/n057/pdf/57011.pdf

TEJEDOR, F. J. (Dir.) (1998). Las estrategias utilizadas por los profesores universitarios para la evaluación del aprendizaje de los alumnos. Informe de Investigación. Salamanca: CIDE.

TEJEDOR, F. J. (2003). Un modelo de evaluación del profesorado universitario. Revista de Investigación Educativa, 21 (1), 157-182.

TRILLO, F. Y PORTO, M. (2002). La evaluación de los estudiantes en el marco de la evaluación de la calidad de las Universidades. Revista de Educación, 328, 283-301.

WORTHINGTON, A. (2002). The impact of student perceptions and characteristics on teaching evaluations: a case study in finance education. Assessment and Evaluation in Higher Education, 27 (1), 49-64.

ZUO, B. Y RATSOY, E. W. (1999). Student Participation in University Governance. The Canadian Journal of Higher Education, XXIX (1), 1-26.

\section{Correspondencia con los autores}

\section{Rosario ORDÓÑEZ-SIERRA}

Facultad de Ciencias de la Educación

Dpto. de Didáctica y Organización Educativa

C/ Pirotecnica, s/n Campus Ramón y Cajal

41013 Sevilla (España)

e-mail: rordonez@us.es

Margarita R. RODRÍGUEZ-GALLEGO

Universidad de Sevilla

Facultad de Ciencias de la Educación

Dpto. de Didáctica y Organización Educativa

C/ Pirotecnica, s/n Campus Ramón y Cajal

41013 Sevilla (España)

e-mail: margaguez@us.es 